\title{
New readers start here
}

\section{Derek Richards}

Editor, Evidence-based Dentistry
With the launch of our new format for UK members of the British Dental Association, in which the journal is integral to the British Dental Journal, it is worth revisiting how we obtain the core content of Evidence-based Dentistry.

EBD Editors regularly search Medline, using a number of standard search strategies (see example, Table 1). As of June 2009, Medline lists 35938 journals, of which 5376 are currently indexed. This includes 165 dental journals, from Acta Odontológica Latinoamericana to Zhonghua kou qiang yi xue za zhi (Chinese Journal of Stomatology). The focus of our searches is systematic reviews and randomised controlled trials, although other study designs (such as cohort and case-control studies) are also considered. Identified studies are assessed for inclusion in the journal based on their methodology and their relevance for practitioners.

The number of potential dental systematic review articles have increased by about $44 \%$ since $E B D$ was first launched in 1998 (Table 1). Many of the reviews identified by this search strategy, however, will not be systematic reviews and, of those, many are not conducted to the high standards recommended by the Cochrane Collaboration. Consequently EBD adopts a more pragmatic and inclusive approach to the selection of systematic reviews for the journal. Randomised controlled trials and other study designs are also assessed on methodology and relevance to practitioner.

\section{Table 1. PubMed systematic review search strategy}

\section{Search strategy}

((dental caries) OR ("Stomatognathic Diseases"[MeSH]) OR ("Mouth Neoplasms"[MeSH]) OR (leukoplakia) OR (periodontal) OR (denture) OR ("Dental Prosthesis"[MeSH]) OR (Prosthodontic) OR (Endodontic) OR ("Endodontics"[MeSH]) OR ("Prosthodontics"[MeSH]) OR (dental pulp disease) OR ("Dental Pulp

Diseases" [MeSH] OR "Periapical Diseases"[MeSH]) OR (Dental Restoration) OR ("Dentistry"[MeSH]) OR (dentistry) OR (oral surgery) OR ("Surgery, Oral"[MeSH])

OR (oral pathology) OR ("Pathology, Oral"[MeSH]) OR (oral medicine) OR

("Oral Medicine"[MeSH]) OR (Oral radiology) OR ("Diagnosis, Oral"[MeSH]) OR (bitewing) OR (Oral Health promotion) OR (TOOTH) OR (DENTAL AMALGAM)) AND ((systematic[sb]) OR (meta-analysis) OR (metaanalysis) OR (systematic review))

\begin{tabular}{l|l}
\hline Limits from 1998/01/01 to $1998 / 12 / 31$ & 2137 \\
\hline Limits from 2008/01/01 to 2008/12/31 & 3080 \\
\hline
\end{tabular}

Once a paper has been identified and selected, a structured abstract is prepared using a standard structure by our team of abstractors. Following this, we then identify a reviewer to prepare a commentary on the review. We invite the reviewer to address some of the major points (good or bad) of the article in a constructive manner, putting it into the context of other relevant literature in the field. We also ask reviewers to identify the relevance for the general practitioner and to identify a maximum of two key practice points that we could include as bullet points at the end of the commentary.

The idea behind these commentaries is to bring to practitioners' attention the good-quality information that might pass them by. Our new format provides the full reference and contact details of the original authors of the papers for which we prepare commentaries, at the top of the page. We encourage readers whose interest is sparked by our commentaries to seek out the original paper to see if their view of it concords with our reviewer's. If you would like to raise issues about the commentary itself we also encourage you to write to the journal: we do consider correspondence for publication on our letters page.

In addition to the commentaries on published papers, which are the core content of the EBD, we also welcome original submissions related to the practice of evidence-based dentistry, DEBTS (Dental Evidence-based Topics $)^{1}$ and Toolbox articles. 1. Richards D. Creating a DEBT. Evid Based Dent 2007;
8: $35-36$.

Evidence-Based Dentistry (2009) 10, 34. doi: $10.1038 /$ sj.ebd.6400640 